\title{
Vitamins D3 and D2 have marked but different global effects on gene expression in a rat oligodendrocyte precursor cell line
}

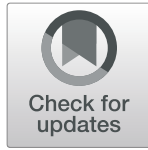

Manuela Mengozzi ${ }^{1,2}$, Andrew Hesketh ${ }^{2,3}$, Giselda Bucca ${ }^{2,3}$, Pietro Ghezzi ${ }^{1,2^{*}}$ and Colin P. Smith ${ }^{2,3}$

\begin{abstract}
Background: Vitamin D deficiency increases the risk of developing multiple sclerosis (MS) but it is unclear whether vitamin D supplementation improves the clinical course of MS, and there is uncertainty about the dose and form of vitamin D (D2 or D3) to be used. The mechanisms underlying the effects of vitamin D in MS are not clear. Vitamin D3 increases the rate of differentiation of primary oligodendrocyte precursor cells (OPCs), suggesting that it might help remyelination in addition to modulating the immune response. Here we analyzed the transcriptome of differentiating rat CG4 OPCs treated with vitamin D2 or with vitamin D3 at $24 \mathrm{~h}$ and $72 \mathrm{~h}$ following onset of differentiation.
\end{abstract}

Methods: Gene expression in differentiating CG4 cells in response to vitamin D2 or D3 was quantified using Agilent DNA microarrays ( $n=4$ replicates), and the transcriptome data were processed and analysed using the $R$ software environment. Differential expression between the experimental conditions was determined using LIMMA, applying the Benjamini and Hochberg multiple testing correction to $p$-values, and significant genes were grouped into coexpression clusters by hierarchical clustering. The functional significance of gene groups was explored by pathway enrichment analysis using the clusterProfiler package.

Results: Differentiation alone changed the expression of about $10 \%$ of the genes at $72 \mathrm{~h}$ compared to $24 \mathrm{~h}$. Vitamin D2 and D3 exerted different effects on gene expression, with D3 influencing 1272 genes and D2 574 at $24 \mathrm{~h}$. The expression of the vast majority of these genes was either not changed in differentiating cells not exposed to vitamin $D$ or followed the same trajectory as the latter. D3-repressed genes were enriched for Gene Ontology (GO) categories including transcription factors and the Notch pathway, while D3-induced genes were enriched for the Ras pathway.

Conclusions: This study shows that vitamin D3, compared with D2, changes the expression of a larger number of genes in OLs. Identification of genes affected by D3 in OLs should help to identify mechanisms mediating its action in MS.

Keywords: Myelination, Multiple sclerosis, Microarrays, Cell differentiation, Central glia-4

\section{Background}

There is evidence that vitamin D deficiency is associated with increased risk of developing multiple sclerosis (MS) and with an increased rate of disease progression (Hayes \& Nashold, 2018; Sintzel et al., 2018). However, the

\footnotetext{
*Correspondence: P.Ghezzi@bsms.ac.uk; p.ghezzi@bsms.ac.uk

${ }^{1}$ Department of Clinical and Experimental Medicine, Brighton and Sussex

Medical School, Brighton BN1 9PS, UK

${ }^{2}$ Centre for Regenerative Medicine and Devices, University of Brighton,

Brighton BN2 4GJ, UK

Full list of author information is available at the end of the article
}

therapeutic efficacy of vitamin D supplementation in MS patients is still an open question, and interventional trials have reported inconclusive results (Jagannath et al., 2018).

Vitamin D is produced in the skin by the action of ultraviolet light. After two successive hydroxylations, the $1,25(\mathrm{OH}) \mathrm{D}$ active form binds to the intracellular vitamin $\mathrm{D}$ receptor (VDR) and, in complex with the retinoid $\mathrm{X}$ receptor (RXR), regulates hundreds of genes through the vitamin D response element (VDRE) (Hayes \& Nashold, 2018; Bikle, 2014).

(c) The Author(s). 2020 Open Access This article is licensed under a Creative Commons Attribution 4.0 International License, which permits use, sharing, adaptation, distribution and reproduction in any medium or format, as long as you give appropriate credit to the original author(s) and the source, provide a link to the Creative Commons licence, and indicate if changes were made. The images or other third party material in this article are included in the article's Creative Commons licence, unless indicated otherwise in a credit line to the material. If material is not included in the article's Creative Commons licence and your intended use is not permitted by statutory regulation or exceeds the permitted use, you will need to obtain permission directly from the copyright holder. To view a copy of this licence, visit http://creativecommons.org/licenses/by/4.0/. 
Vitamin D can also be assimilated from the diet or as a supplement. Both the plant/fungus-derived vitamin D2, ergocalciferol, and the animal-derived D3 form, cholecalciferol, are available as nutritional supplements; both can be hydroxylated into their active forms and bind VDR with similar affinity (Hollis, 1984), but there are differences in their catabolism and in their binding affinity to vitamin $\mathrm{D}$ binding protein (DBP), the major vitamin $\mathrm{D}$ transport protein in blood, with vitamin D2 binding DBP with lower affinity and being catabolised more quickly (Hayes \& Nashold, 2018; Bikle, 2014).

Studies comparing vitamin D2 and D3 for their ability to raise and maintain circulating levels of $25(\mathrm{OH}) \mathrm{D}$, the serum marker of systemic vitamin D levels, have shown that vitamin D2 is less effective than D3 when given as single bolus (Tripkovic et al., 2012); however, results following daily administration of D2 or D3 are controversial, with clinical trials showing higher efficacy of D3 (Zittermann et al., 2014; Tripkovic et al., 2017) or equal efficacy (Holick et al., 2008). In a recent metaanalysis, vitamin D2 and D3 were found equally effective in raising vitamin $D$ levels in infants (Zittermann et al., 2020). It has been suggested that vitamin D2 supplementation reduces circulating vitamin D3 levels; however, a recent randomized controlled trial showed that the reciprocal is also true (Hammami et al., 2019). The general consensus is that vitamin D3 is the preferred form to be administered (Jagannath et al., 2018), but few studies have compared D2 and D3 in terms of their direct cellular effects under controlled conditions.

The mechanisms mediating the protective action of vitamin D in MS are not fully characterized. In addition to regulating calcium homeostasis, vitamin D is immunomodulatory and anti-inflammatory (Hayes \& Nashold, 2018; Sintzel et al., 2018; Mora et al., 2008). In the last twenty years, many studies have reported effects on the brain; vitamin D inhibits neuroinflammation, is neuroprotective and neurotrophic (Hayes \& Nashold, 2018; Garcion et al., 2002; Eyles et al., 2013; Cui et al., 2015). VDR is present in neurons and glial cells (Baas et al., 2000; Eyles et al., 2005). Vitamin D acts directly on brain cells by promoting the differentiation of neural stem cells (NSCs) into neurons and oligodendrocytes (OLs), and inducing the expression of growth factors, such as nerve growth factor (Ngf), in neurons and OLs (Baas et al., 2000; Shirazi et al., 2015). The regenerative and neurotrophic effects of vitamin $D$, together with the presence of VDR in OLs, suggest that it might have a myelinating action, which might contribute to its protective effects in MS. In this respect, de la Fuente et al. found that vitamin D increased the differentiation and maturation of primary oligodendrocyte progenitor cells (OPCs) (de la Fuente et al., 2015).

Rat central glia-4 (CG4) OPCs are considered a good model of myelination in vitro; the cells are maintained at the precursor stage by culture with growth factors (GFs), and can be differentiated into OLs by withdrawal of GFs and culture in differentiation medium (DM) (Louis et al., 1992; Stariha \& Kim, 2001; Gyetvai et al., 2017). Interestingly, Baas et al. found that CG4 cells express VDR, as primary OPCs, and respond to vitamin D by increasing expression of VDR and of Ngf; however, an effect on myelin proteins was not found, leading to the conclusion that vitamin $\mathrm{D}$ does not positively affect the differentiation of these cells (Baas et al., 2000).

To better understand the molecular mechanisms by which vitamin D acts on OLs, we analyzed the gene expression profile of rat CG4 OPCs in response to vitamin $\mathrm{D}$, and compared vitamin D2 with D3.

\section{Methods \\ Cell culture}

The rat CG4 OPCs, originally obtained from primary cultures of bipotential oligodendrocyte-type-2-astrocytes (O-2A) (Louis et al., 1992), were cultured as previously reported (Gyetvai et al., 2017; Cervellini et al., 2013). CG4 cells are maintained at the precursor stage by culture in growth medium (GM), consisting of Dulbecco's modified Eagle medium (DMEM; Sigma-Aldrich) supplemented with biotin $(10 \mathrm{ng} / \mathrm{ml})$, basic fibroblast growth factor (bFGF; 5 $\mathrm{ng} / \mathrm{ml}$ ), platelet-derived growth factor (PDGF; $1 \mathrm{ng} /$ $\mathrm{ml}$ ), N1 supplement (all from Sigma-Aldrich) and $30 \%$ B104-conditioned medium, obtained as previously reported (Cervellini et al., 2013). The cells can be differentiated into mature OLs by withdrawal of growth factors (bFGF and PDGF) and of B104conditioned medium and culture in DM, consisting of DMEM-F12 (Invitrogen) supplemented with progesterone $(3 \mathrm{ng} / \mathrm{ml})$, putrescine $(5 \mu \mathrm{g} / \mathrm{ml})$, sodium selenite $(4 \mathrm{ng} / \mathrm{ml})$, insulin $(12.5 \mu \mathrm{g} / \mathrm{ml})$, transferrin $(50 \mu \mathrm{g} / \mathrm{ml})$, biotin $(10 \mathrm{ng} / \mathrm{ml})$, thyroxine $(0.4 \mu \mathrm{g} / \mathrm{ml})$ and glucose $(3 \mathrm{~g} / \mathrm{l})$ (all from Sigma-Aldrich).

Cells were plated in poly-L-ornithine-coated 12-well plates (100,000 cells in $2 \mathrm{ml}$ GM per well). After overnight culture, the cells were induced to differentiate by switching to DM. Cells were cultured for the indicated times; half of the medium was changed every other day. For vitamin D treatment, vitamin D2 (1,25-dihydroxyvitamin D2; Cayman Chemical) or vitamin D3 (1,25-dihydroxyvitamin D3; Sigma-Aldrich) were dissolved in ethanol (96\%; Sigma-Aldrich) at $100 \mu \mathrm{M}$ and then 
diluted in medium at the final concentration (100 nM). Vehicle (ethanol $0.1 \%$ ) was added to untreated cells.

\section{RNA extraction}

Each sample was lysed with $1 \mathrm{ml}$ of QIAzol (QIAGEN). Total RNA was extracted using the miRNeasy system and protocol (QIAGEN). RNA concentration, purity and integrity were determined using a NanoDrop 1C (NanoDrop Technologies) spectrophotometer and an Agilent 4200 TapeStation (Agilent Technologies). All samples had a A260/A280 ratio $>1.9$ and RNA Integrity Number $>8.2$.

\section{RT-qPCR}

Reverse transcription (RT) and real time quantitative PCR (qPCR) were carried out as reported (Gyetvai et al., 2017), using TaqMan ${ }^{\circ}$ gene expression assays (Applied Biosystems/Thermo Fisher Scientific). Results were normalized to Hprt1 expression (reference gene) and expressed as fold change (FC) versus one of the control samples (as indicated), chosen as the calibrator. The Taqman $^{\circ}$ assays used were: Rn00569647_m1 (Jag1); Rn00586568_m1 (Dll3); Rn00569763_m1 (Psen1); Rn01508945_g1 (Rras2); Rn01533872_m1 (Ngf); Rn00676060_m1 (Tgfb2); Rn01450612_s1 (Sox4); Rn00690431_m1 (Tfeb); Rn00690616_m1 (VDR); Rn01527840_m1 (Hprt1).

\section{Microarrays}

Biological quadruplicate samples were analyzed for each treatment group. In total, 24 arrays were analyzed: 4 untreated, 4 vitamin D2 and 4 vitamin D3 at each time point (following, respectively, $24 \mathrm{~h}$ and $72 \mathrm{~h}$ of treatment). Total RNA (200 ng) was amplified by in vitro transcription using the Low Input Quick Amp One color kit, incorporating Cy3-labelled CTP (Agilent Technologies), and hybridized onto SurePrint G3 Rat GE $8 \times 60 \mathrm{~K}$ Microarrays v2 (AMADID 074036; Agilent Technologies) for $16-20 \mathrm{~h}$ at $65^{\circ} \mathrm{C}$ in an Agilent oven with rotisserie. Following hybridization, the arrays were washed and scanned to derive the array images, using an Agilent microarray scanner G2505C. Feature Extraction Software (v11.5; Agilent) was used to generate the array data from the images, using Agilent grid 074036_D_F_20181024.

\section{Microarray data analysis}

Data analysis was performed in $\mathrm{R}$ (Team, 2014) using the LIMMA package (Ritchie et al., 2015). Grid annotation for the AMADID 074036 microarray was downloaded from Agilent eArray, and gene annotations were obtained from the Rattus.norvegicus $\mathrm{R}$ package based on the rn5 genome build (Team, 2015). Microarray data were background corrected using the "normexp" method (with an offset of 50) and quantile normalized, producing normalized expression values in the log base 2 scale. Data were then filtered to remove all control probes, and non-control probes exhibiting low signals across the array sets. Keeping only those non-control probes that were at least $10 \%$ brighter than negative control probe signals on at least four arrays (based on the existence of four replicates per condition) left 31,691 out of 62,976 probes. Data from identical replicate probes within the same array were then averaged to produce expression values at the unique probe level, obtaining 20, 326 unique probes, of which 15,247 are assigned to genome features with an Entrez gene identifier (Entrez ID).

Testing for differential expression between the experimental conditions was performed in LIMMA using linear modeling. Significance $p$-values were corrected for multiplicity using the Benjamini and Hochberg (BH) method, obtaining adjusted p-values (adj. P.Val). All the 20,326 unique probes were subjected to differential expression analysis but only the data for the significantly differentially expressed probes mapping to an Entrez ID (5565) are reported in Additional File 1 and were considered in the downstream analyses.

Hierarchical clustering was performed by complete linkage clustering and using the Pearson correlation for the distance metric. Abundance values for each probe were transformed to z-scores for clustering. Functional enrichment analysis was performed using the $\mathrm{R}$ package clusterProfiler (version 3.8.1) (Yu et al., 2012). The software produces adjusted $p$-values (p.adjust) using the $\mathrm{BH}$ correction method.

\section{Results}

\section{Set up and experimental treatment}

We first established the appropriate experimental conditions for studying the effect of vitamin D on the gene expression profile of differentiating CG4 cells. In a previous study, Baas et al. found that VDR is present in CG4 OPCs and its levels are increased 2-fold after culture for two days in differentiating conditions (Baas et al., 2000); we therefore hypothesized that a preincubation in DM for two days before treatment with vitamin $\mathrm{D}$ might increase the basal levels of VDR and the response to vitamin D. Since VDR is induced by vitamin D3 (Baas et al., 2000), we monitored basal and vitamin D3-induced VDR levels in different culture conditions. The dose of $100 \mathrm{nM}$ vitamin D3 was chosen based on previous studies (Baas et al., 2000; de la Fuente et al., 2015), having verified that a higher dose of D3 (200 nM) did not induce higher levels of VDR expression compared to D3 $100 \mathrm{nM}$, as assessed at $72 \mathrm{~h}$ of D3 treatment (FC vs control, mean $\pm \mathrm{SD}, N=3$; D3 at $100 \mathrm{nM}$ : $2.3 \pm 0.2$; D3 at $200 \mathrm{nM}: 2.0 \pm 0.3)$.

Cells were plated, left to adhere overnight, then medium was changed to DM and cells were treated 
with vitamin D3 added at the same time as DM (Fig. 1a) or after pre-incubation in DM for $48 \mathrm{~h}$ (Fig. 1b). VDR mRNA was measured after $24 \mathrm{~h}$ or $72 \mathrm{~h}$ of incubation with vitamin D3 in both conditions. A 48$\mathrm{h}$ pre-incubation in DM increased basal levels of VDR and vitamin D3-induced VDR levels at $24 \mathrm{~h}$ and $72 \mathrm{~h}$ (Fig. 1). Of note, VDR expression was induced by vitamin D3 at $24 \mathrm{~h}$ only after cells were predifferentiated for $48 \mathrm{~h}$ (Fig. 1b, left panel). At this time point, vitamin D2 at the same dose $(100 \mathrm{nM})$ induced similar levels of VDR as D3 (FC vs control, mean $\pm \mathrm{SD}, N=3$; D3: $2.8 \pm 1.2$; D2: $2.5 \pm 0.7)$. Therefore, the experimental design in Fig. 1b was used for the transcriptome analysis, with vitamin D2 and D3 both used at $100 \mathrm{nM}$.

After $48 \mathrm{~h}$ of pre-differentiation in DM, cells were treated with either vitamin D2 or D3; RNA was isolated following incubation for a further $24 \mathrm{~h}$ and $72 \mathrm{~h}$ and analyzed using whole genome DNA microarrays. The DNA microarray design uses unique oligonucleotide probes to quantify the abundance of gene transcripts containing the complementary sequences, and can include more than one probe per gene. In the presentation of the results below we make the distinction between probes and genes when necessary, and occasionally use the term "probes-genes" when no distinction is required.

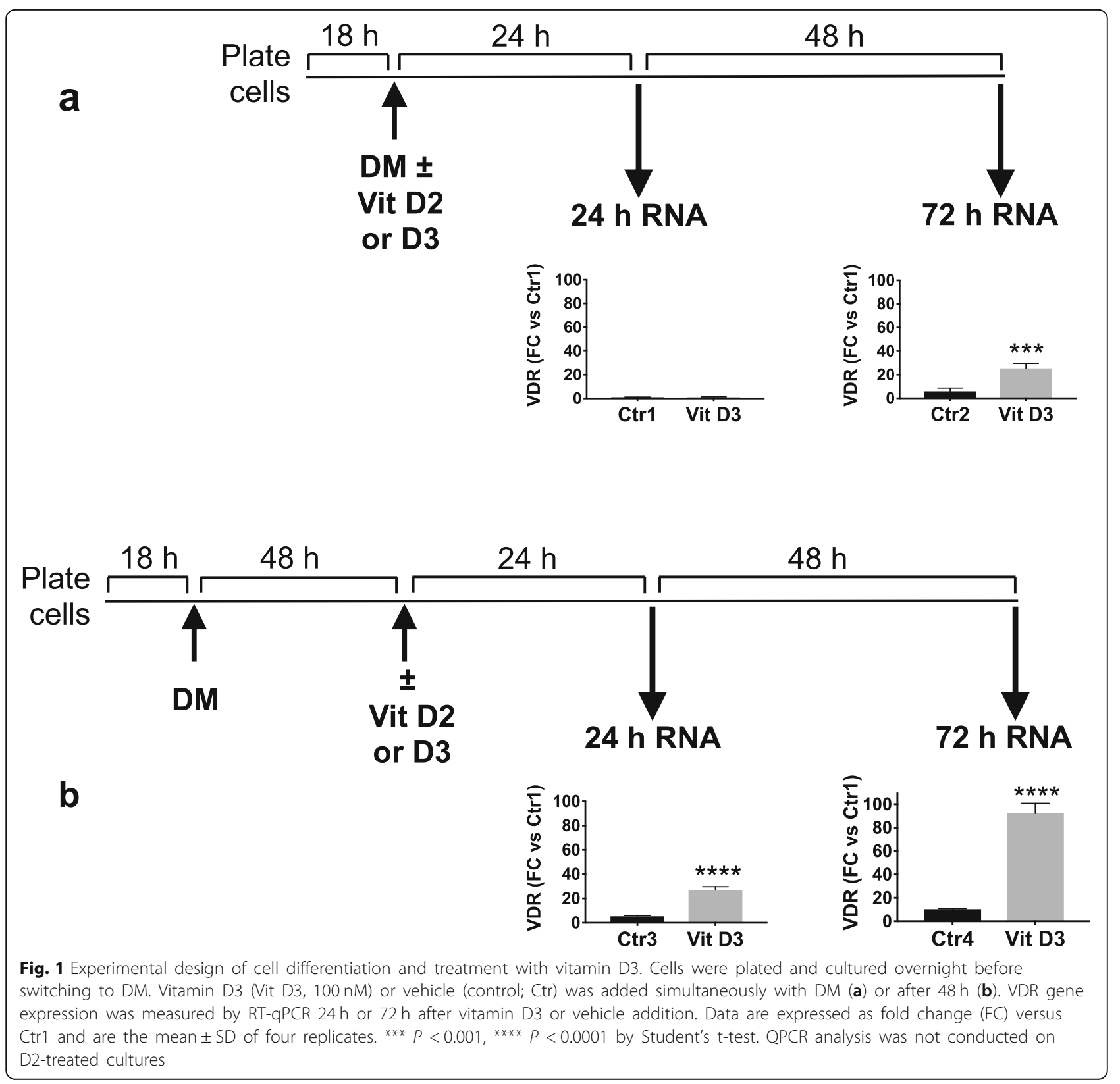


Effect of vitamin D2 and D3 on gene expression profile Overall, addition of either vitamin D2 or D3 to differentiating cells significantly affected gene expression, an effect that was more marked at $72 \mathrm{~h}$ (Fig. 2). Out of 15,247 probes, corresponding to 12,261 genes (ArrayExpress Accession number E-MTAB-8098) about 30\% were affected by either vitamin D2 or D3 at $72 \mathrm{~h}$, and $10 \%$ at $24 \mathrm{~h}$ (Fig. 2a).

The numbers of probes-genes affected by vitamin D2 and D3 were broadly similar at $72 \mathrm{~h}$, with most differentially expressed genes regulated by both D2 and D3 (Fig. 2a, b). However, the shorter-term response at $24 \mathrm{~h}$ showed a more pronounced response to vitamin D3, with notably larger numbers of probes-genes down-regulated compared with vitamin D2 (721 versus 147 probes, corresponding to 664 versus 139 genes respectively; Fig. 2a). At $24 \mathrm{~h}$, most genes regulated by $\mathrm{D} 2$ were also regulated by $\mathrm{D} 3$, with some other genes regulated by D3 only (Fig. 2b).

To visualize the global effect of vitamin D2 and D3 in relation to untreated cells, we performed a hierarchical cluster analysis of the 5565 probes differentially regulated when comparing vitamin D2 or D3 with their respective controls at both time points, as well as untreated controls at $72 \mathrm{~h}$ versus $24 \mathrm{~h}$. The results are shown as a heatmap in Fig. 2c.

By comparing untreated control cells (Ctrl) at $72 \mathrm{~h}$ with Ctrl at $24 \mathrm{~h}$, we can see that differentiation alone noticeably affects gene expression. The actual number of probes-genes regulated in Ctrl $72 \mathrm{~h}$ versus $\mathrm{Ctrl} 24 \mathrm{~h}$ is reported in Fig. 2a. Figure 2c also shows that a number of genes with low expression in control cells are upregulated by both vitamin D2 and D3 at $24 \mathrm{~h}$, an effect even more marked than that of differentiation at $72 \mathrm{~h}$ (e.g. cluster 5 in Fig. 2c). Likewise, some of the genes that have a high expression in $24 \mathrm{~h}$ Ctrl cells seem to be down-regulated by vitamin D2 and D3 at $24 \mathrm{~h}$, an effect similar to differentiation alone (e.g. cluster 9 in Fig. 2c). This trend is confirmed at $72 \mathrm{~h}$, where vitamin D2 and D3 have a more marked effect than at $24 \mathrm{~h}$.

\section{Effect of differentiation}

To analyze the effect of vitamin D in relation to the effect of differentiation, we focused on the genes changed

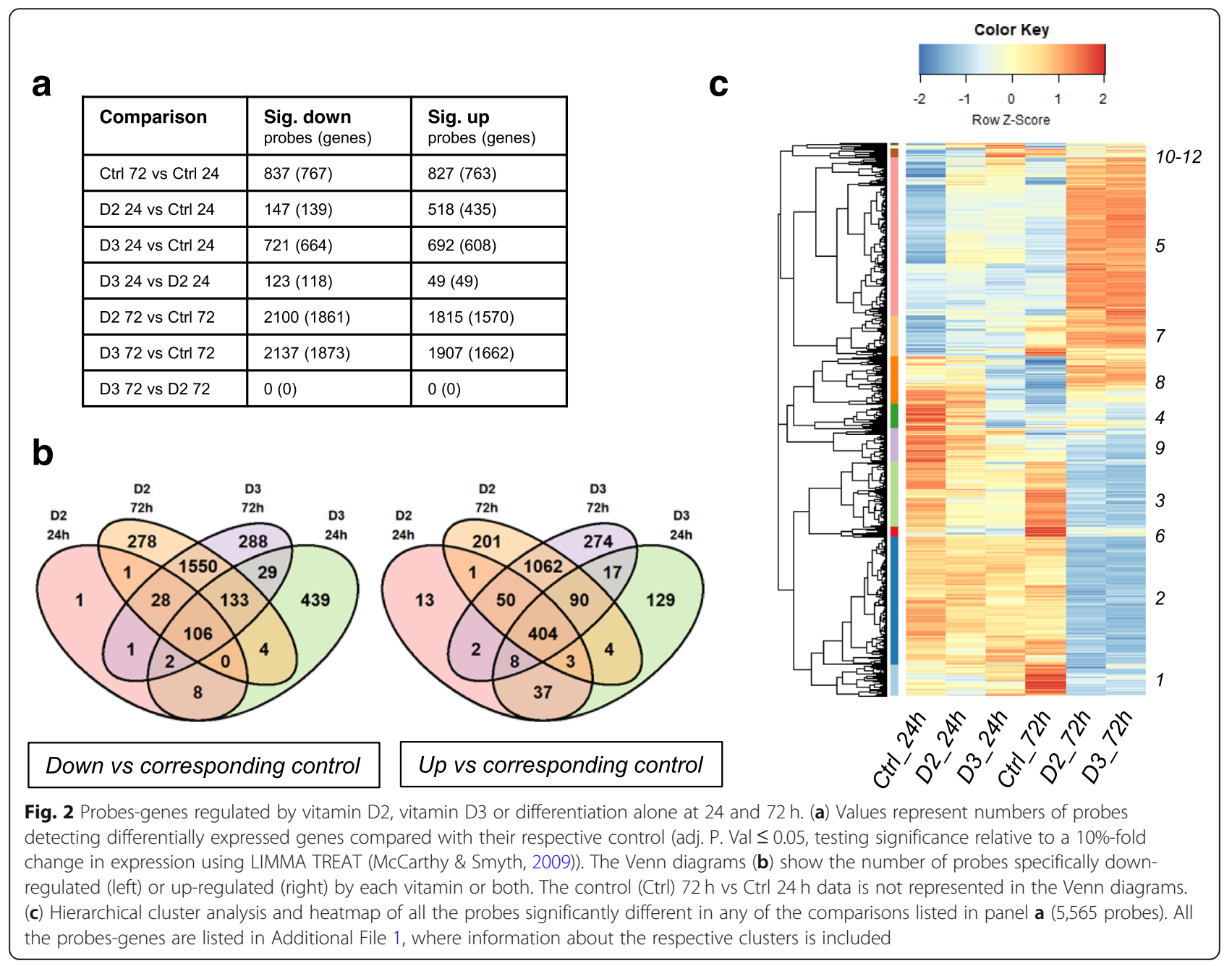


by culture in DM alone. As reported in Fig. 2a, 837 probes (767 genes) were down-regulated and 827 (763 genes) were up-regulated in untreated cells (Ctrl) at $72 \mathrm{~h}$ compared to $\mathrm{Ctrl}$ at $24 \mathrm{~h}$.

The functional enrichment analysis of the 767 down-regulated and of the 763 up-regulated genes in the $72 \mathrm{~h}$ versus $24 \mathrm{~h}$ control cells is reported in Fig. 3a. This reveals that most of the down-regulated genes were associated with the cell cycle and the upregulated genes were significantly (p.adjust $\leq 0.05$ ) enriched for Gene Ontology Biological Process (GOBP) categories including "cell maturation" (p.adjust 7.77E-03), "axon ensheathment" (p.adjust 2.28E-03), "myelination" (p.adjust 6.75E-03); this is consistent with the fact that CG4 cells in DM differentiate over time (Gyetvai et al., 2017; Cervellini et al., 2013).

To visualize the effect of vitamin D2 and D3 at 24h on the genes down-regulated or up-regulated by differentiation alone (767 and 763 respectively, as mentioned above), we performed a hierarchical cluster analysis (Fig. 3b) of the expression abundance of the corresponding probes (837 and 827 respectively) in untreated cells at 24 and $72 \mathrm{~h}$ (highlighting the effect of differentiation alone) and in vitamin D2- and D3-treated cells at $24 \mathrm{~h}$ (highlighting the effect of each vitamin, that can be visually compared to the effect of differentiation alone).

The clusters 1-4 in Fig. 3b comprise all the probes corresponding to the genes increased by differentiation alone. Both vitamins, and in particular vitamin D3, preferentially increase the expression of these genes at $24 \mathrm{~h}$.

The same trend was confirmed for the downregulated genes (clusters $5-8$ in Fig. 3b), where the preferential downregulating effect of vitamin D3 compared to D2 was more marked. Functional enrichment

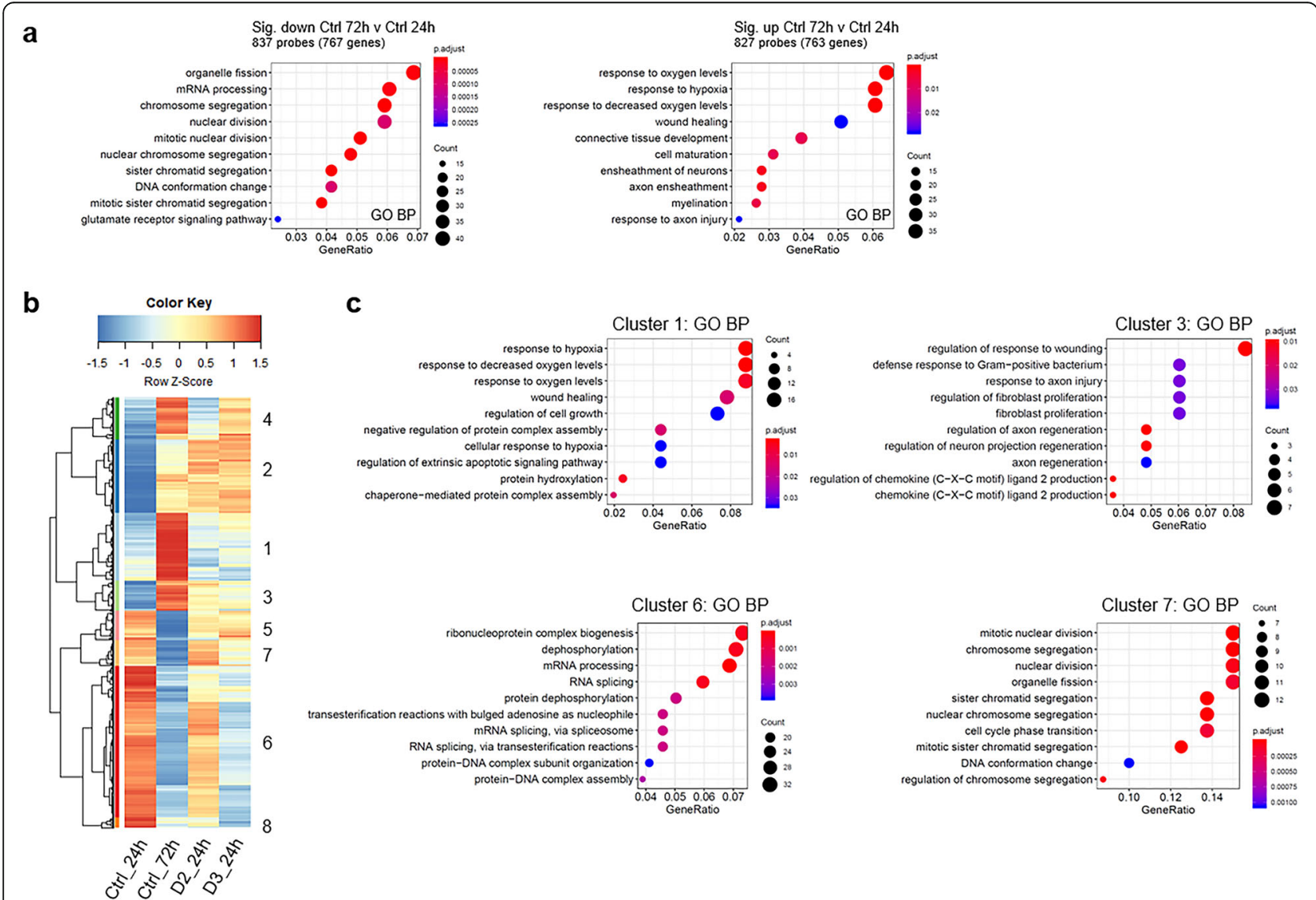

Fig. 3 Effect of vitamin D2 and D3 on the genes regulated by differentiation: functional enrichment analysis. (a) The enriched Gene Ontology Biological Processes (GO-BPs) among the 767 down-regulated (left) and 763 up-regulated (right) genes, shown in Fig. 2a as "Ctrl 72 vs Ctrl 24 ", are listed. The size of the dots is proportional to the number of genes and the color represents the corrected $p$-value (p.adjust), as indicated in the legend. (b) Cluster analysis and heat map of the expression data for the probes corresponding to the genes in (a) (837 down-regulated and 827 up-regulated by differentiation alone, Fig. 2a); the expression signal at $24 \mathrm{~h}$ and $72 \mathrm{~h}$ in untreated cells and at $24 \mathrm{~h}$ in D2- and D3-treated cells is shown, to visualize the effect of D2 and D3 versus their control at $24 \mathrm{~h}$ and the effect of differentiation alone at $72 \mathrm{~h}$ versus $24 \mathrm{~h}$. (c) Enriched GO$\mathrm{BP}$ terms corresponding to some of the clusters. All the probes-genes included in the clusters and in the GO-BPs are listed in Additional File 2 
analysis of the down-regulated clusters 6 and 7 (Fig. 3c) identified GO-BP categories associated with cell cycle, whereas "axon regeneration" (p.adjust 3.73E-02) was one of the GO-BP terms significantly enriched among the clusters increased by differentiation and by vitamin D2 and D3 (cluster 3, Fig. 3c).

\section{Analysis of vitamin D-regulated genes in relation to differentiation}

A more specific analysis of the effects of vitamin D on gene expression in relation to the change in the gene expression profile induced by differentiation alone (Ctrl $72 \mathrm{~h}$ versus Ctrl 24h) is shown in Fig. 4.

More than half of the genes up-regulated (in orange in Fig. 4) at $24 \mathrm{~h}$ by vitamin D2 (a) or D3 (b) were also induced by differentiation (in orange in the bottom pie-charts of the two panels), with the remaining genes (in grey in the bottom pie-charts of the panels) not affected by differentiation alone. Only one gene (spondin 1, Additional File 1) induced by vitamin D2 or D3 at $24 \mathrm{~h}$ was changed in the opposite direction by differentiation.

Of the genes that were down-regulated at $24 \mathrm{~h}$ (in blue in Fig. 4) by vitamin D2 (a) or D3 (b), most genes were down-regulated (in blue in the bottom pie charts) or unaffected (in grey in the bottom pie charts) by differentiation. Only few vitamin D-down-regulated probes (six for D2 and eleven for D3) were changed in the opposite direction by differentiation (Additional File 1). In summary, a 24-h exposure to vitamin D had either a unique

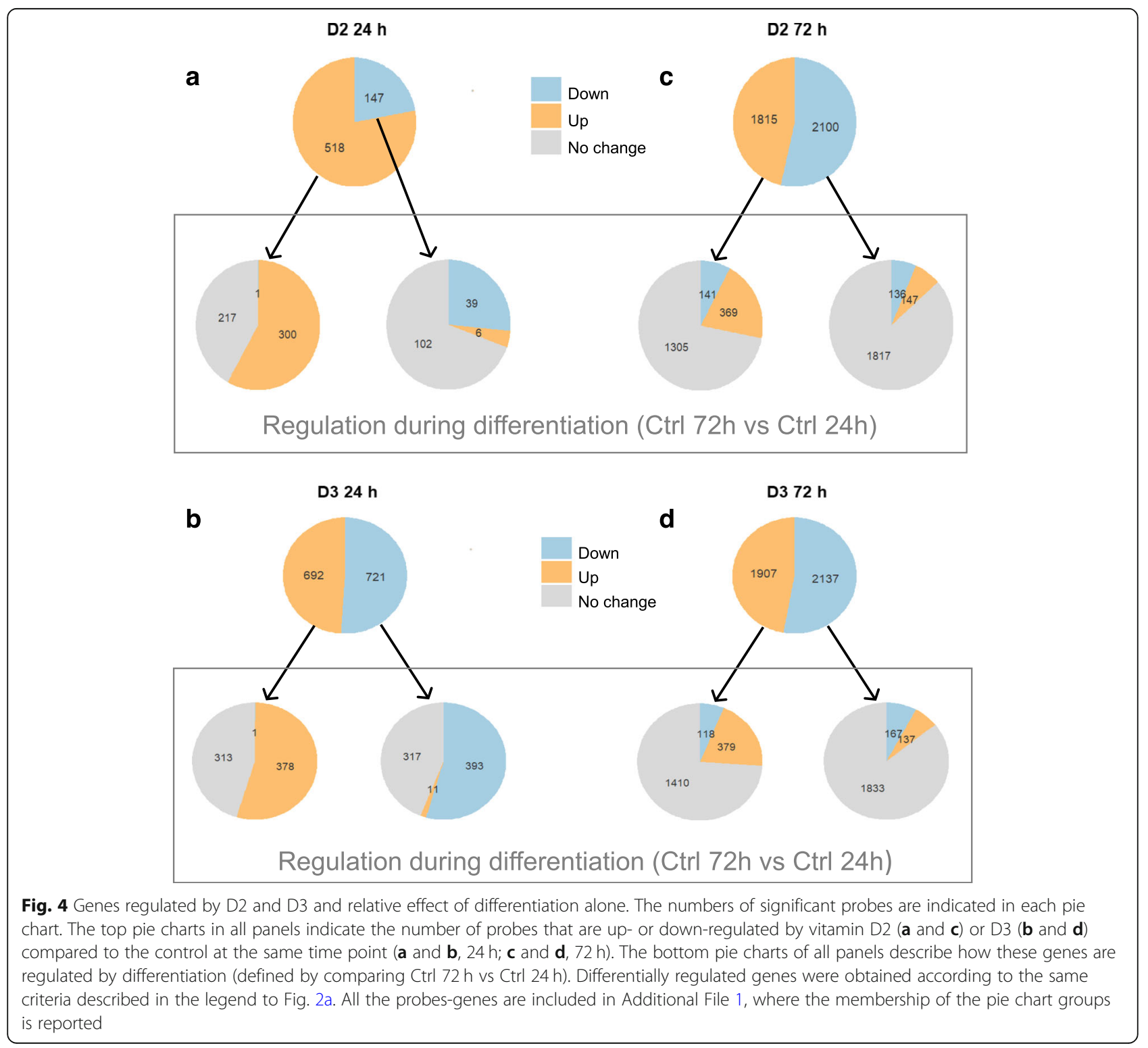


effect for specific genes or an effect on gene expression similar to that of differentiation.

A similar analysis was performed at $72 \mathrm{~h}$ (Fig. $4 \mathrm{c}$ and d). At this time point, the overlap between vitamin $\mathrm{D}$ and differentiation-regulated genes was smaller, and most of the effect on the gene expression profile was unique to vitamin $\mathrm{D}$.

\section{Genes uniquely regulated by vitamin D3 and not by D2}

Because vitamin D3 had a more marked effect on gene expression at $24 \mathrm{~h}$ than D2 (Fig. 2a, Fig. 4a and b), we analyzed the genes uniquely regulated by D3 (but not by D2) at $24 \mathrm{~h}$ and their behavior in relation to differentiation. As shown in Fig. 5, all the genes uniquely regulated by vitamin D3 were either not changed by differentiation in absence of vitamin $\mathrm{D}$, or followed the same trajectory as differentiation, with only five probes down-regulated by D3 but up-regulated by differentiation.

\section{Gene expression profile in oligodendrocyte cells is more greatly affected by vitamin D3 than D2}

To analyze the differential effect on gene expression of vitamin D3 compared with D2, we focused on the probes-genes uniquely regulated by vitamin D3 (and not by vitamin D2) at $24 \mathrm{~h}$. At this time point, vitamin D3 uniquely down-regulated 605 probes and up-regulated 240 probes, as shown in Fig. 5.

Functional enrichment analysis of the genes corresponding to the probes significantly decreasing in abundance after $24 \mathrm{~h}$ in response to either vitamin D2

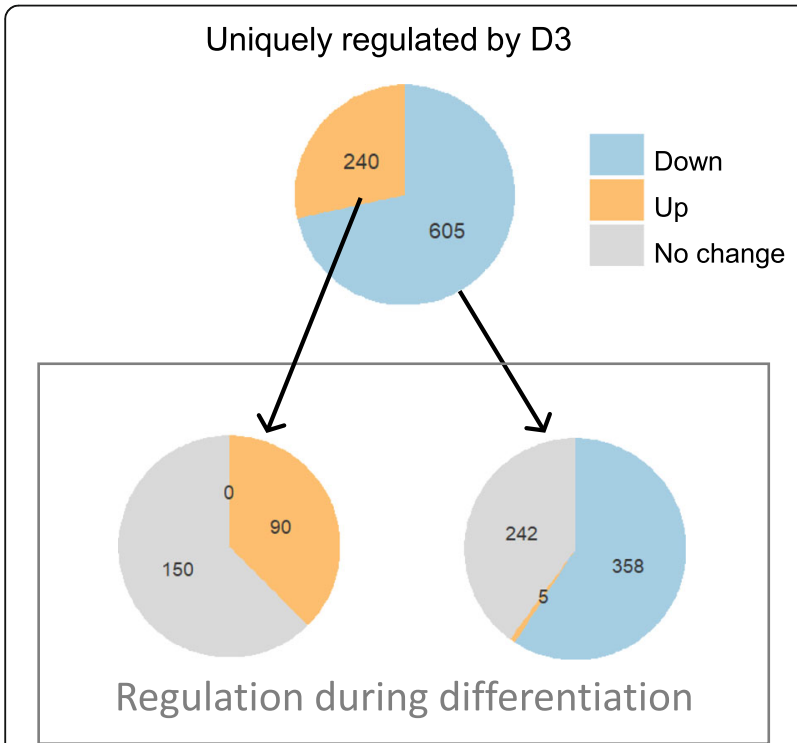

Fig. 5 Genes uniquely regulated by vitamin D3 at $24 \mathrm{~h}$ and relative effect of differentiation alone. Numbers in the top pie charts refer to probes up-regulated (orange) or down-regulated (blue) by D3 versus control but not by D2 versus control. The list of the probes-genes is reported in Additional File 1 $(n=147$, Fig. $2 \mathrm{a}$ and 4a) or D3 $(n=721$, Fig. $2 \mathrm{a}$ and 4b) and of those uniquely down-regulated by D3 ( $n=605$; Fig. 5$)$ indicated that the two forms of vitamin D produce notably different early effects on oligodendrocyte cells (Additional File 3: Supplementary Fig. S1, Additional File 4). Interestingly, the 605 genes uniquely down-regulated by D3 at $24 \mathrm{~h}$ were significantly enriched for GO terms associated with transcription factor activity ("transcription factor complex", p.adjust 3.59E-03; "transcription factor activity", p.adjust 8.29E-04) suggesting that the two forms of vitamin $\mathrm{D}$ might exert different effects on transcriptional regulation by these complexes. Other functions similarly and potentially more adversely affected by D3 compared to D2 at $24 \mathrm{~h}$ included "RNA splicing" (p.adjust 3.07E-02), "axonogenesis" (p.adjust 7.73E-03), Toll-like receptor (TLR) cascade pathways (e.g. "TLR3 cascade", p.adjust 4.01E-03), "MAPK signaling pathways" (p.adjust 2.25E-02) and "Notch signaling pathway" (p.adjust 2.71E-02).

Functional enrichment analysis of the genes corresponding to the probes significantly increased at $24 \mathrm{~h}$ by vitamin D2 ( $n=518$, Fig. $2 \mathrm{a}$ and $4 \mathrm{a})$ or D3 ( $n=692$; Fig. $2 \mathrm{a}$ and $4 \mathrm{~b}$ ) and of those uniquely up-regulated by D3 $(n=240$, Fig. 5$)$ is reported in Additional File 3: Supplementary Fig. S2 and Additional File 4. The genes uniquely up-regulated by D3 were enriched for the GO term "GTP binding" (p.adjust 4.11E-02) whereas analysis of all the genes up-regulated by vitamin D3 showed an enrichment of the Ras signal transduction pathway ("Ras protein signal transduction", p.adjust 3.46E-02).

\section{RT-qPCR validation}

The significant expression changes observed in VDR and in key genes of interest from the Notch (Jag1, Dll3, Psen1) and Ras (RRas2, Ngf, Tgfb2) signaling pathways, and in the transcription factors Sox 4 and Tfeb, were verified using RT-qPCR. Eight of the nine significant changes investigated were successfully verified (Additional File 5: Supplementary Table S1).

\section{Discussion}

The main finding of this study is that vitamin D2 and D3 exert markedly different effects on gene expression in differentiating OPCs. Although there is some overlap in the genes they regulate, D3 affects a larger repertoire of genes. In particular, we identified some genes that are specifically influenced by D3 only (Fig. 5 and Additional File 1), showing that, in this experimental model, D2 and D3 (or their respective derivatives) might behave as distinct molecular entities with different biological activities.

In vivo, studies where D2 and D3 are compared are likely to mainly reflect differences in their pharmacokinetics. This should not be an issue in an in vitro model 
that should reflect the direct effect of the molecule tested, and our results are likely to relate to differences in the biological activity of the two forms of vitamin D. Although, reportedly, vitamin D2 and D3 have similar affinities for VDR (Bikle, 2014; Hollis, 1984), they may have different affinity for other vitamin $\mathrm{D}$ binding proteins. In fact, the action of vitamin $\mathrm{D}$ is mediated by other receptors and binding proteins such as the retinoid-related orphan receptors (RORs) and Erp57, a membrane steroid-binding protein related to protein disulfide isomerase (Khanal \& Nemere, 2007; Nemere et al., 2010; Slominski et al., 2017). These additional receptors may have important biological roles in the central nervous system, and the protective effect of D3 on axonal growth has been linked to activation of membrane-associated rapid response steroid-binding receptor (MARRS)/Erp57 (Tohda et al., 2012).

Vitamin D3 and D2 both up-regulated VDR expression (Additional File 1), which was also upregulated during OPC differentiation, in agreement with a previous report (Baas et al., 2000). Both vitamins regulated the expression of many genes that were either not changed in differentiating cells not exposed to vitamin D or followed the same trajectory as the latter (Figs. 4 and 5); only very few genes followed the opposite expression trajectory, suggesting that in this system, as in primary OLs (de la Fuente et al., 2015), vitamin D promotes rather than antagonizing differentiation.

Although at $72 \mathrm{~h}$ both vitamin D2 and D3 had similar effects on gene expression, at $24 \mathrm{~h}$ vitamin D3 was more potent, changing the expression of a substantially higher number of genes. The preferential action of D3 versus D2 was particularly evident when considering the downregulated genes; only about one fourth of the genes down-regulated by D3 were also down-regulated by D2 at $24 \mathrm{~h}$, whereas most of the genes down-regulated by D2 were also down-regulated by D3, as shown in Fig. 2. The higher efficacy of vitamin D3 compared to D2 in increasing myelination had been previously reported in a rat model of peripheral nerve injury (Chabas et al., 2013), but no studies had directly compared vitamin D2 and D3 in vitro on OL differentiation.

Functional enrichment analysis of the genes uniquely down-regulated by D3 (unchanged by D2) at $24 \mathrm{~h}$ highlighted an over-representation of the GO Molecular Function (GO-MF) "transcription factor activity" (Additional File 3: Supplementary Fig. S1); among these, SRY-related HMG-Box Gene 4 (Sox4) and transcription factor EB (Tfeb) (Additional File 4), whose high expression has been previously associated with inhibition of myelination (Potzner et al., 2007; Meireles et al., 2018). In addition, the KEGG MAPK signaling pathway was overrepresented; of note, we previously reported that in this model inhibition of ERK1/2 MAPK increases OL differentiation (Gyetvai et al., 2017). Fibroblast growth factor receptor 2 (Fgfr2) and platelet derived growth factor A (Pdgfa) were included in this pathway (Additional File 4); since both Fgf and Pdgf are needed to maintain CG4 OPCs in the undifferentiated state (Louis et al., 1992; Gyetvai et al., 2017), inhibition of Fgfr and Pdgf would promote differentiation.

Considering all the genes down-regulated by D3 at $24 \mathrm{~h}$ (and therefore possibly also by D2), enrichment of the Notch signaling pathway was identified (Additional File 3: Supplementary Fig. S1). Activation of the Notch pathway inhibits OPC differentiation during development and is involved in the limited remyelination that characterizes MS (Wang et al., 1998; John et al., 2002). Notch is a transmembrane receptor that is activated upon binding to several ligands, including members of the Jagged and Deltalike families; these ligands induce sequential cleavage of Notch, with an essential cleavage performed by a gammasecretase, which contains presenilin (Hori et al., 2013). The ligands Jagged 1 (Jag1) and delta-like canonical Notch ligand 3 (Dll3) were inhibited by both vitamin D2 and D3 (Additional File 1). The inhibitory effect of vitamin D3 on gamma-secretase activity had been previously reported in the context of Alzheimer's disease (Grimm et al., 2017); in this respect, gamma-secretase, in addition to Notch, also cleaves the amyloid precursor protein (APP) to produce amyloid-beta. Inhibition of Notch ligands and of other genes belonging to the Notch pathway (Additional File 4) suggests a differentiating effect of vitamin $\mathrm{D}$ on these cells. Interestingly, inhibition of Notch-signalling molecules, which play a role in cancer stem cell maintenance, is one of the mechanisms that mediate the anticancer properties of vitamin D (So \& Suh, 2015; Shan et al., 2017).

Functional enrichment analysis of all the genes upregulated by D3 revealed overrepresentation of the GOBP "Ras protein signal transduction" (Additional File 3: Supplementary Fig. S2 and Additional File 4). GTPases of the Ras family affect cell survival, proliferation and differentiation (Crespo \& Leon, 2000), and among these Ras related 2 (Rras2) was recently found to be essential for OL differentiation (Sanz-Rodriguez et al., 2018). Included in this pathway are also Ngf and transforming growth factor beta 2 (Tgfb2), both reported to activate Ras-dependent signalling pathways and to promote OPC differentiation (Marei et al., 2013; Schmitz et al., 2010; McKinnon et al., 1993; Palazuelos et al., 2014; Li et al., 2017). Vitamin D3-induced upregulation of Ngf in these cells was previously reported (Baas et al., 2000). Of note, Rras2 and Ngf were also induced by D2, whereas Tgfb2 was uniquely induced by D3 (Additional File 4). Also included in this pathway were several Rab genes, such as Rab36, Rab9d, Rab6a, and Rab3d. Rab proteins, involved in vesicular transport, are up-regulated during $\mathrm{OL}$ 
differentiation, in particular when OL start synthesizing myelin (Bouverat et al., 2000); interestingly Rab6a and Rab3d were uniquely up-regulated by D3 (GO-MF "GTP binding", Additional File 3: Supplementary Fig. S2; Addition File 4). In addition, vitamin D3-induced transcription factors (GO Cellular Component (GO-CC) "nuclear transcription factor complex", Additional File 3: Supplementary Fig. S2, Additional File 4) included Sox2, whose role in OL differentiation was recently reported (Kuhbandner, 2018; Zhang et al., 2018) and CCAAT/enhancer binding protein beta (Cebpb), a VDR-responsive gene that controls the differentiation of myeloid leukaemia cells (Marchwicka \& Marcinkowska, 2018).

Although we did not observe any direct effect of vitamin D on the expression of myelin genes, the induction or repression effect of vitamin $\mathrm{D}$, and in particular of vitamin D3, on genes positively or negatively involved in OL differentiation and myelination confirms the myelinating effects of vitamin D in OLs that have been previously reported (de la Fuente et al., 2015; Gomez-Pinedo et al., 2020). The lack of a direct effect of vitamin D on the expression of myelin genes in these cells is in agreement with previous studies (Baas et al., 2000) and highlights a limitation of this experimental model.

\section{Conclusions}

The identification of specific vitamin D3-regulated genes in OPCs could provide important insights to understand the mechanisms mediating the activity of vitamin $\mathrm{D}$ in demyelinating disease and point to mechanisms additional to those associated with immune regulation, the latter being a major research focus for the action of vitamin D in the context of MS (Hayes \& Nashold, 2018). However, to completely understand the physiological relevance of our results, our findings should be confirmed at the protein level and in other experimental models, including in vivo models of myelination.

\section{Supplementary information}

Supplementary information accompanies this paper at https://doi.org/10. 1186/s10020-020-00153-7.

Additional file 1. Summary of all significant differences identified in the study (only microarray probes annotated with an Entrez gene identifier are considered). A complete description of the data reported is provided in the README sheet (xIsx 1.8 MB)

Additional file 2. Functional enrichment analysis (Gene Ontology, Biological Process category) of the genes with an Entrez gene identifier represented by the probes clustered in Fig. 3. The description of the data reported is provided in the README sheet (xIsx $38 \mathrm{~KB}$ ).

Additional file 3: Supplementary Fig. S1. Functional enrichment analysis of the genes down-regulated by vitamin D. Supplementary Fig. S2. Functional enrichment analysis of the genes up-regulated by vitamin D (PDF 935 KB).
Additional file 4. Comparative functional enrichment analysis of the three groups of genes in Additional File 3: Supplementary Fig. S1 and S2. The description of the data reported is provided in the README sheet (xlsx $46 \mathrm{~KB})$.

Additional file 5: Supplementary Table S1. RT-qPCR validation of microarray data (PDF 29 KB).

\section{Abbreviations}

bFGF: basic fibroblast growth factor; BH: Benjamini and Hochberg; CG4: rat central glia-4; DBP: vitamin D binding protein; DII3: delta-like canonical Notch ligand 3; DM: differentiation medium; DMEM: Dulbecco's modified Eagle medium; GFs: growth factors; GM: growth medium; GO-BP: Gene Ontology Biological Process; GO-CC: Gene Ontology Cellular Component; GO-MF: Gene Ontology Molecular Function; Hprt1: hypoxanthine phosphoribosyltransferase 1; Jag1: Jagged 1; MS: multiple sclerosis; Ngf: nerve growth factor; OLs: oligodendrocytes; OPCs: oligodendrocyte precursor cells; PDGF: platelet-derived growth factor; Psen1: presenilin 1; qPCR: quantitative PCR; Rras2: Ras related 2; RT: reverse transcription; Sox2: SRY-related HMG-Box Gene 2; Sox4: SRY-related HMG-Box Gene 4; Tfeb: transcription factor EB; Tgfb2: transforming growth factor beta 2; VDR: vitamin D receptor

\section{Acknowledgements}

We gratefully acknowledge the financial support from Prof Matteo Santin of the Centre for Regenerative Medicine and Devices (CRMD), University of Brighton.

\section{Authors' contributions}

CPS and PG conceived the study and supervised the project, MM and GB carried out the experiments, $\mathrm{AH}$ analyzed the results, MM, AH and PG wrote the manuscript although all the authors contributed to data analysis and to elaboration of the figures and of the final manuscript. The authors read and approved the final manuscript.

\section{Funding}

Supported by the Centre for Regenerative Medicine and Devices (CRMD), University of Brighton.

\section{Availability of data and materials}

The datasets supporting the conclusions of this article are available in ArrayExpress (www.ebi.ac.uk/arrayexpress/) with Accession number E-MTAB8098.

\section{Ethics approval and consent to participate}

Not applicable.

\section{Consent for publication}

Not applicable.

Competing interests

The authors declare they have no competing interests.

\section{Author details}

'Department of Clinical and Experimental Medicine, Brighton and Sussex Medical School, Brighton BN1 9PS, UK. ${ }^{2}$ Centre for Regenerative Medicine and Devices, University of Brighton, Brighton BN2 4GJ, UK. ${ }^{3}$ School of Pharmacy and Biomolecular Sciences, University of Brighton, Brighton BN2 $4 G J, U K$.

Received: 25 October 2019 Accepted: 26 February 2020 Published online: 09 April 2020

\section{References}

Baas $D$, et al. Rat oligodendrocytes express the vitamin $D(3)$ receptor and respond to 1,25-dihydroxyvitamin D(3). Glia. 2000;31:59-68.

Bikle DD. Vitamin D metabolism, mechanism of action, and clinical applications. Chem Biol. 2014;21:319-29.

Bouverat BP, Krueger WH, Coetzee T, Bansal R, Pfeiffer SE. Expression of rab GTPbinding proteins during oligodendrocyte differentiation in culture. J Neurosci Res. 2000;59:446-53. 
Cervellini I, et al. Erythropoietin (EPO) increases myelin gene expression in CG4 oligodendrocyte cells through the classical EPO receptor. Mol Med. 2013;19: 223-9.

Chabas JF, et al. Cholecalciferol (vitamin D(3)) improves myelination and recovery after nerve injury. PLoS One. 2013;8:e65034.

Crespo P, Leon J. Ras proteins in the control of the cell cycle and cell differentiation. Cell Mol Life Sci. 2000;57:1613-36.

Cui $X$, et al. Vitamin D and the brain: key questions for future research. J Steroid Biochem Mol Biol. 2015;148:305-9.

de la Fuente $A G$, et al. Vitamin $D$ receptor-retinoid $X$ receptor heterodimer signaling regulates oligodendrocyte progenitor cell differentiation. J Cell Biol. 2015;211:975-85.

Eyles DW, Burne TH, McGrath JJ. Vitamin D, effects on brain development, adult brain function and the links between low levels of vitamin D and neuropsychiatric disease. Front Neuroendocrinol. 2013;34:47-64.

Eyles DW, Smith S, Kinobe R, Hewison M, McGrath JJ. Distribution of the vitamin $D$ receptor and 1 alpha-hydroxylase in human brain. J Chem Neuroanat. 2005;29:21-30

Garcion E, Wion-Barbot N, Montero-Menei CN, Berger F, Wion D. New clues about vitamin D functions in the nervous system. Trends Endocrinol Metab. 2002;13:100-5.

Gomez-Pinedo U, et al. Vitamin D increases remyelination by promoting oligodendrocyte lineage differentiation. Brain Behav. 2020;10:e01498.

Grimm MOW, et al. Vitamin D and its analogues decrease amyloid-beta (Abeta) formation and increase Abeta-degradation. Int J Mol Sci. 2017:18.

Gyetvai G, et al. Erythropoietin increases myelination in Oligodendrocytes: gene expression profiling reveals early induction of genes involved in lipid transport and metabolism. Front Immunol. 2017:8:1394.

Hammami MM, Abuhdeeb K, Hammami S, Yusuf A. Vitamin-D2 treatmentassociated decrease in $25(\mathrm{OH}) \mathrm{D} 3$ level is a reciprocal phenomenon: a randomized controlled trial. BMC Endocr Disord. 2019;19:8.

Hayes CE, Nashold FE. (2018) Vitamin D and multiple sclerosis. In: Vitamin D, Volume 2: Health, Disease and Therapeutics, Fourth Edition. Feldman D (ed.) Elsevier Inc., pp. 989-1024

Holick MF, et al. Vitamin D2 is as effective as vitamin D3 in maintaining circulating concentrations of 25-hydroxyvitamin D. J Clin Endocrinol Metab. 2008;93:677-81.

Hollis BW. Comparison of equilibrium and disequilibrium assay conditions for ergocalciferol, cholecalciferol and their major metabolites. J Steroid Biochem. 1984;21:81-6.

Hori K, Sen A, Artavanis-Tsakonas S. Notch signaling at a glance. J Cell Sci. 2013; 126:2135-40.

Jagannath VA, et al. (2018) Vitamin D for the management of multiple sclerosis. Cochrane Database Syst Rev 9: Cd008422.

John GR, et al. Multiple sclerosis: re-expression of a developmental pathway that restricts oligodendrocyte maturation. Nat Med. 2002:8:1115-21.

Khanal RC, Nemere I. The ERp57/GRp58/1,25D3-MARRS receptor: multiple functional roles in diverse cell systems. Curr Med Chem. 2007;14:1087-93.

Kuhbandner K. Uncovering the role of Sox2 in oligodendroglia. J Neurosci. 2018; 38:4460-1.

Li S, GU X, Yi S. The regulatory effects of transforming growth factor-beta on nerve regeneration. Cell Transplant. 2017;26:381-94.

Louis JC, Magal E, Muir D, Manthorpe M, Varon S. CG-4, a new bipotential glial cell line from rat brain, is capable of differentiating in vitro into either mature oligodendrocytes or type-2 astrocytes. J Neurosci Res. 1992;31:193-204.

Marchwicka A, Marcinkowska E. Regulation of expression of CEBP genes by variably expressed vitamin $\mathrm{D}$ receptor and retinoic acid receptor alpha in human acute myeloid leukemia cell lines. Int J Mol Sci. 2018;19.

Marei $\mathrm{HE}$, et al. Over-expression of hNGF in adult human olfactory bulb neural stem cells promotes cell growth and oligodendrocytic differentiation. PLoS One. 2013;8:e82206.

McCarthy DJ, Smyth GK. Testing significance relative to a fold-change threshold is a TREAT. Bioinformatics. 2009;25:765-71.

McKinnon RD, Piras G, Ida JA Jr, Dubois-Dalcq M. A role for TGF-beta in oligodendrocyte differentiation. J Cell Biol. 1993;121:1397-407.

Meireles AM, et al. (2018) The Lysosomal transcription factor TFEB represses myelination downstream of the rag-Ragulator complex. Dev. Cell 47: 319 330.e315.

Mora JR, Iwata M, von Andrian UH. Vitamin effects on the immune system: vitamins a and D take Centre stage. Nat Rev Immunol. 2008;8:685-98.
Nemere I, Garbi N, Hammerling GJ, Khanal RC. Intestinal cell calcium uptake and the targeted knockout of the 1,25D3-MARRS (membrane-associated, rapid response steroid-binding) receptor/PDIA3/Erp57. J Biol Chem. 2010;285:31859-66.

Palazuelos J, Klingener M, Aguirre A. TGFbeta signaling regulates the timing of CNS myelination by modulating oligodendrocyte progenitor cell cycle exit through SMAD3/4/Fox01/Sp1. J Neurosci. 2014;34:7917-30.

Potzner MR, et al. Prolonged Sox4 expression in oligodendrocytes interferes with normal myelination in the central nervous system. Mol Cell Biol. 2007;27: 5316-26.

Ritchie ME, et al. (2015) Limma powers differential expression analyses for RNAsequencing and microarray studies. Nucleic Acids Res. 43: e47.

Sanz-Rodriguez M, et al. R-Ras1 and R-Ras2 are essential for Oligodendrocyte differentiation and survival for correct myelination in the central nervous system. J Neurosci. 2018;38:5096-110.

Schmitz M, et al. Mutual effects of caveolin and nerve growth factor signaling in pig oligodendrocytes. J Neurosci Res. 2010;88:572-88.

Shan $\mathrm{NL}$, et al. Vitamin D compounds inhibit cancer stem-like cells and induce differentiation in triple negative breast cancer. J Steroid Biochem Mol Biol. 2017;173:122-9.

Shirazi HA, Rasouli J, Ciric B, Rostami A, Zhang GX. 1,25-Dihydroxyvitamin D3 enhances neural stem cell proliferation and oligodendrocyte differentiation. Exp Mol Pathol. 2015:98:240-5.

Sintzel MB, Rametta M, Reder AT. Vitamin D and multiple sclerosis: a comprehensive review. Neurol Ther. 2018;7:59-85.

Slominski AT, et al. Endogenously produced nonclassical vitamin D hydroxymetabolites act as "biased" agonists on VDR and inverse agonists on RORalpha and RORgamma. J Steroid Biochem Mol Biol. 2017;173:42-56.

So JY, Suh N. Targeting cancer stem cells in solid tumors by vitamin D. J Steroid Biochem Mol Biol. 2015;148:79-85.

Stariha RL, Kim SU. Mitogen-activated protein kinase signalling in oligodendrocytes: a comparison of primary cultures and CG-4. Int J Dev Neurosci. 2001;19:427-37.

Team BC. (2015) Rattus.Norvegicus: annotation package for the Rattus.Norvegicus object. R package version 1.3.1.

Team RC. R: a language and environment for statistical computing. Vienna, Austria: R Foundation for statistical computing; 2014.

Tohda C, Urano T, Umezaki M, Nemere I, Kuboyama T. Diosgenin is an exogenous activator of 1,25D(3)-MARRS/Pdia3/ERp57 and improves Alzheimer's disease pathologies in 5XFAD mice. Sci Rep. 2012;2:535.

Tripkovic $L$, et al. Comparison of vitamin D2 and vitamin D3 supplementation in raising serum 25-hydroxyvitamin D status: a systematic review and metaanalysis. Am J Clin Nutr. 2012;95:1357-64.

Tripkovic L, et al. Daily supplementation with 15 mug vitamin D2 compared with vitamin D3 to increase wintertime 25-hydroxyvitamin D status in healthy south Asian and white European women: a 12-wk randomized, placebocontrolled food-fortification trial. Am J Clin Nutr. 2017;106:481-90.

Wang $\mathrm{S}$, et al. Notch receptor activation inhibits oligodendrocyte differentiation. Neuron. 1998:21:63-75.

Yu G, Wang LG, Han Y, He QY. clusterProfiler: an R package for comparing biological themes among gene clusters. OMICS. 2012;16:284-7.

Zhang S, et al. Sox2 is essential for Oligodendroglial proliferation and differentiation during postnatal brain myelination and CNS Remyelination. J Neurosci. 2018;38:1802-20.

Zittermann A, Ernst JB, Gummert JF, Borgermann J. Vitamin D supplementation, body weight and human serum 25 -hydroxyvitamin $D$ response: a systematic review. Eur J Nutr. 2014;53:367-74.

Zittermann A, Pilz S, Berthold HK. Serum 25-hydroxyvitamin D response to vitamin D supplementation in infants: a systematic review and meta-analysis of clinical intervention trials. Eur J Nutr. 2020;59:359-69.

\section{Publisher's Note}

Springer Nature remains neutral with regard to jurisdictional claims in published maps and institutional affiliations. 